and automobiles these alloys have proved invaluable. In the United States of America railway carriage frames are being constructed of duralumin, thus diminishing the dead weight, as compared with the net weight carried, very considerably. Die castings of intricate shapes, such as gear wheels, are now being made, to a remarkable degree of accuracy, of aluminium bronzes-alloys of copper and aluminium, containing more than 80 per cent. of copper.

The rapidity with which these alloys have been developed and have won the confidence of engineers is a tribute to the careful scientific work that has been done in connexion with their constitution and physical properties. The success achieved by the aluminium alloys has encouraged a number of workers to investigate the possibilities of alloying magnesium, which has only a specific gravity of 1.7 as compared with 2.67 for aluminium, with other metals, and already some success has been achieved in the application of magnesium alloys, containing more than 90 per cent. of magnesium, to the manu- facture of high-speed pistons for internal combustion engines and other purposes.

Sir Henry Fowler paid a tribute to the important research work done in the great works, at the National Physical Laboratory, and also at the universities, but he emphasised the necessity for researches conducted jointly by men of science and engineers. Unfortunately, he did not suggest any method whereby the ability at present in the universities, which owing to pressure of teaching and other routine duties is not able, except at the cost of health, to apply itself to the solution of many problems with which industry is faced, can be given the necessary time and means to carry out research. The only hope seems a more generous provision of funds for the direct assistance of research in the engineering and metallurgicaldepart. ments of the universities, in order that the condition of the apt quotation with which the address concluded may be fulfilled: "The wisdom of the scribe cometh by the opportunity of leisure; and he that hath little business shall become wise.",

\title{
Scientific Aspects of Intense Magnetic Fields and High Voltages. ${ }^{1}$
}

By Sir Ernest Rutherford, O.M., Pres.R.S.

$I^{\mathrm{N}}$ $\mathrm{N}$ the past gur laboratories have had to be content with the comparatively weak magnetic fields provided by the ordinary electro-magnets and the voltages supplied by simple electrostatic machines and induction coils. In order to push further our investigations in many directions, much stronger magnetic fields and higher voltages are required in the laboratory. Scientific men thus naturally follow with great interest advances in these directions, whether undertaken for purely scientific or for technical uses.

By means of modern electrostatic machines it is not difficult to produce weak direct currents at potentials from 200,000 to 300,000 volts, while a large well-insulated induction coil can give momentary voltages of a similar magnitude. The wide use of $X$-rays for diagnostic and therapeutic purposes has led to a marked improvement in apparatus for exciting intense $X$-rays. The requirement of very penetrating $X$-rays for deep therapy in our hospitals has led to the construction of comparatively light transformers, which will supply the requisite small currents at voltages between 300,000 and 500,000 .

One of the simplest ways of producing very high voltages is by the Tesla transformer, in which the oscillatory discharge of a Leyden jar is passed through the primary of an air transformer. In this way it is not difficult to produce voltages in the secondary of the order of a million volts, and I understand as much as five million volts have been obtained in the Carnegie Institution of Washington. The striking effects produced by these rapidly oscillating discharges from a Tesla coil, and the immunity with which long sparks may be taken through the body, are well known to all. The rapid frequency of the oscillations and the compara-

1 From the presidential address delivered at the anniversary meeting of the Royal Society on Nov. 30 . tively small energy given to the secondary of a Tesla coil has, however, restricted its use for general technical purposes as a source of high voltages, although it is now finding an application for the testing of insulating materials.

In order to transmit electrical power economically over long distances, there is a continuous tendency to raise the voltage in the transmission lines. This increase of the operating voltage has led to the need of very high voltages to test the insulating properties of these lines and their transformers and the effect of electric surges in them. In the course of the last few years a number of high-voltage plants have been installed for testing purposes in various countries, which give from one to two million volts. These voltages may be obtained either by a very large well-insulated power transformer or more generally by a cascade method employing several transformers in which the secondary current of one transformer passes through the primary of a second, and so on, the cores of the successive transformers being mounted on insulating pedestals. This cascade method is very advantageous for the purpose, since it allows a great reduction in weight and dimensions of the transformers. Such a high-tension plant in full operation is a striking sight, giving a torrent of sparks several yards in length and resembling a rapid succession of lightning flashes on a small scale. Actually the highest voltage so far obtained by these methods is very small compared with the voltage in a normal lightning flash from a cloud to the earth, where the difference of potential may be as high as a thousand million volts.

There appears to be no obvious limit to the voltages obtainable by the cascade arrangement of transformers, except that of expense and the size of the building required to install them. I am informed that the General Electric Company of

No. 3031, VoL. 120] 
Schenectady has a working plant giving $2,800,000$ volts (max.), and hopes soon to have ready a plant to give 6 million volts.

While no doubt the development of such high voltages serves a useful technical purpose, from the purely scientific point of view interest is mainly centred on the application of these high potentials to vacuum tubes in order to obtain a copious supply of high-speed electrons and high-speed atoms. So far, we have not yet succeeded in approaching, much less surpassing, the success of the radioactive elements, in providing us with high-speed $a$-particles and swift electrons. The a-particle from radium $C$ is liberated with an encrgy of $7 \cdot 6$ million electron volts, that is, it has the energy acquired by an electron in a vacuum which has fallen through this difference of potential. 'The swiftest $\beta$-rays from radium have an energy of about 3 million electron volts, while a voltage of more than 2 million would be required to produce $X$-rays of the penetrating power of the $\gamma$-rays.

The application of high voltages to vacuum tubes presents serious technical problems, but a vigorous attack on this side of the question has been recently undertaken by Dr. Coolidge. In 1894, Lenard made the discovery that high-speed cathode rays generated in a discharge tube could be transmitted into the open air through a very thin window, and made very important observations on the laws of absorption of these swift particles. The voltage used to acceleratc the clectrons in these experiments seldom exceeded 80,000 volts and the rays were stopped in passing through a few inches of air. Taking advantage of the great improvements in vacuum technique and the ease of supply of electrons from a glowing filament, Dr. Coolidge has constructed an electron tube which will stand 300,000 volts, the rays passing into the air through a thin plate of chrome-nickel-iron alloy about 0.0005 inch thick.

It has not so far been found practicable to apply much more than 300,000 volts to a single tube, on account of the danger of a flash over, due possibly to the pulling-out of electrons from the cathode by the intense electric field. For the application of still higher voltages, a number of tubes are arranged in series and communicating with one another, the fall of potential in each being about 300,000 volts. In these preliminary experiments, a large induction coil has been uscd to generate the voltage. So far, experiments have been made with three tubes in series and 900,000 volts, giving a supply of electrons corresponding to one or two milliamperes through the thin window in the last tube. This gives an intense beam of high-velocity elcctrons, which spreads out into a hemisphere, due to the scattering of the clectrons in passing through the metal window and the surrounding air, extending to a distance of about two metres from the window. Marked luminous effects are produced in the air itself and in phosphorescent bodies placed in the path of the rays. I am informed by Dr. Coolidge that further experiments are in progress, and it is hoped to extend the system for still higher voltages.
While the energy acquired by the individual electrons in falling through 900,000 volts is smaller than that possessed by the swifter $\beta$-particles expelled from radium, the number cmitted from the electron tube is very much greater; for example, the number of electrons per second corresponding to a current of 2 milliamperes is equivalent to the number of $\beta$-rays cmitted per second from about $150,000 \mathrm{gm}$. of radium in equilibrium.

While important progress has been made in artificially producing streams of swift electrons, there is still much work to be done before we can hope to produce streams of atoms and electrons of a much higher individual energy than the $\alpha$ or $\beta$-particle spontaneously liberated from radioactive bodies. As we have seen, the a-particle from radium $C$ is initially expelled with an energy of about 8 million electron volts. So far, the $\alpha$-particle has the greatest individual energy of any particle known to science, and for this reason it has been invaluable in exploring the inner structure of the atom and giving us important data on the magnitude of the deflecting field in the neighbourhood of atomic nuclei and of the dimensions of the nuclei. In case of some of the lighter atoms, the $\alpha$-particle has sufficient energy to penetrate deeply into the nucleus and to cause its disintegration, manifested by the liberation of swift protons.

It would be of great scientific interest if it were possible in laboratory experiments to have a supply of electrons and atoms of matter in general, of which the individual energy of motion is greater even than that of the $a$-particle. 'This would open up an extraordinarily interesting field of investigation which could not fail to give us information of great value, not only on the constitution and stability of atomic nuclei but also in many other directions.

It has long been my ambition to have available for study a copious supply of atoms and electrons which have an individual energy far transcending that of the $\alpha$ - and $\beta$-particles from radioactive bodies. I am hopeful that I may yet have my wish fulfilled, but it is obvious that many experimental difficulties will have to be surmounted before this can be realised, even on a laboratory scale.

We shall now consider briefly the present situation with regard to the production of intense magnetic fields. Electro-magnets are ordinarily employed for this purpose, and the magnetic fields obtainable are in the main limited by the magnetic saturation of the iron. By the use of large electromagnets and conical pole pieces, the magnetic induction can be concentrated to some extent. For example, in the large Weiss eleotro-magnet, a ficld of 80,000 gauss can be obtained over a volume corresponding to about a pin's head, and a ficld of about 50,000 gauss through a volume of about 20 cubic $\mathrm{mm}$. In general, however, most experiments have been restricted to fields less than 35,000 gauss.

In order to push this method of obtaining

No. 3031, VoL. 120] 
magnetic fields to the practical limit, Prof. Cotton, of Paris, has designed and has under construction a very large electro-magnet. The cross-section of the iron will be of the order of one square metre, and about 500 kilowatts will be required to excite it. Such a large electro-magnet will not give a much stronger maximum field than existing ones, but will produce a field of given intensity through a larger volume. No doubt this electro-magnet will prove very useful in experiments where steady fields of high intensity are required through a reasonable volume.

In order to provide magnetic fields of the order of half a million gauss, the use of the electro-magnet must be abandoned. Some years ago, Dr. Kapitza suggested that intense momentary magnetic fields could be obtained by sending a very strong current through a coil for such a short interval that the heating effect in the coil is restricted to a permissible value. It is well known that momentary currents of great intensity can be produced by the discharge of a large high-voltage condenser through a coil. Experiments of this kind have been made by Dr. Wall, in which the duration of the discharge was of the order of one-thousandth of a second. It is estimated that in this way a field of about 200,000 gauss may be reached.

In his experiments to obtain intense magnetic fields, Dr. Kapitza at first employed a special form of accumulator to send a very strong current through a coil for about one-hundredth of a second, the current if necessary being sharply broken after this interval. In this way it was shown to be practicable to carry out experiments on the Zeeman effect, and in bending a-particles in magnetic fields considerably stronger than those obtainable with ordinary methods. In subsequent experiments, a generator of special design was installed, which gives a very large current, of the order of 70,000 amp., at 2000 volts when short-circuited. A heavy current from the generator is passed for about one-hundredth of a second through a coil and then sharply broken by means of a specially designed automatic break. By this means very strong momentary currents can be produced.

The main difficulty in these experiments has been to construct a coil strong enough to withstand the enormous disrupting forces which arise when a large current is passed through the coil. By special attention to the design, $a$ coil has been made which gives a field of 320,000 gauss over a volume of about 3 c.c. without any signs of fracture. Measurements have been regularly carried out in fields of this magnitude. It is anticipated that the present design of coil will give about 500,000 gauss before bursting, and that still higher fields can be obtained in coils specially constructed for the purpose.

As the current only lasts about one-hundredth of a second, oscillograph methods have to be employed to determine the strength of the current and magnetic field. There seems to be no inherent difficulty in conducting magnetic experiments in these momentary fields, for the shortness of the time available is in many cases compensated for by the magnitude of the effects which arise in such intense fields. The investigations, which have been carried out in the Cavendish Laboratory, have been made possible by the generous support of the Department of Scientific and Industrial Research, which has defrayed the cost of the apparatus and experiments.

The application of these new methods of producing intense fields opens up a wide region of research, where all magnetic properties can be examined in fields ten to twenty times stronger than those hitherto available. Such researches cannot fail to yield results of great interest and importance and to advance our knowledge of magnetic phenomena.

While the application of external magnetic fields of the order of one-million gauss will no doubt markedly perturb the orbits of electrons in the outer structure of the atom, it is not to be anticipated that they will seriously affect the stability of atomic nuclei. General evidence indicates that the magnetic fields within the nucleus are much too great for such a relatively weak external field to cause a disruption of the nucleus. In this direction, the bombardment by high-speed particles is likely to be far more effective than the strongest magnetic field we can hope to generate.

This advance of science depends to a large extent on the development of new technical methods and their application to scientific problems. The recent work to which I have referred, on the development of methods of producing high voltages and intense magnetic fields, is not only of great interest to scientific men in itself but also promises to provide us with more powerful methods of attack on a number of fundamental problems.

\section{News and Views.}

IN February 1925 the residuary trust funds of the estate of the late Dr. Conway Evans, medical officer for the Strand district, who died in 1892, were transferred to the president of the Royal Society and the president of the Royal College of Physicians of London, and their successors in office, that in accordance with the terms of his bequest they " shall apply the same in giving rewards to such person or persons who, in the opinion of the Presidents, have rendcred, or shall from time to time render, some valuable contribution or addition to science as it exists at the time of my death, either by invention, discovery, or other- wise." In accordance with this trust, the president of the Royal Society and the president of the Royal College of Physicians of London have made the first award of the Conway Evans Prize, amounting to 500 guineas, to Sir Charles Sherrington, on the ground that his work on the physiology of the nervous system, and chiefly on the physiology of the brain and spinal cord of the higher animals, has brought many complex nervous functions for the first time within the range of investigation and analysis. His discoveries have had a profound influence throughout the world on the experimental sciences of physiology and

No. 3031, VoL. 120] 